University of Nebraska - Lincoln

DigitalCommons@University of Nebraska - Lincoln

P. F. (Paul Frazer) Williams Publications

Electrical \& Computer Engineering, Department

September 1980

\title{
Electron Densities in Laser-Triggered Hydrogen Sparks
}

S. K. Dhali

Texas Tech University, Lubbock, TX

P. F. Williams

University of Nebraska - Lincoln, pfw@moi.unl.edu

M. A. Gundersen

University of Nebraska - Lincoln

R. J. Crumley

Texas Tech University, Lubbock, TX

Follow this and additional works at: https://digitalcommons.unl.edu/elecengwilliams

Part of the Electrical and Computer Engineering Commons

Dhali, S. K.; Williams, P. F.; Gundersen, M. A.; and Crumley, R. J., "Electron Densities in Laser-Triggered Hydrogen Sparks" (1980). P. F. (Paul Frazer) Williams Publications. 13.

https://digitalcommons.unl.edu/elecengwilliams/13

This Article is brought to you for free and open access by the Electrical \& Computer Engineering, Department of at DigitalCommons@University of Nebraska - Lincoln. It has been accepted for inclusion in P. F. (Paul Frazer) Williams Publications by an authorized administrator of DigitalCommons@University of Nebraska - Lincoln. 


\title{
Electron Densities in Laser-Triggered Hydrogen Sparks
}

\author{
S. K. DHALI, P. F. WILLIAMS, MEMBER, IEEE, R. J. CRUMLEY, AND M. A. GUNDERSEN
}

\begin{abstract}
We have used Stark broadening measurements of the $\mathrm{H}_{\beta}$ emission line to determine the temporally and spatially resolved electron density in laser-triggered hydrogen sparks. In this paper the results of this work are presented and compared with earlier work on conventional overvolted sparks.
\end{abstract}

\section{INTRODUCTION}

$\mathrm{Q}$ UESTIONS concerning the basic physical processes controlling the electrical breakdown of gases are of considerable interest from both engineering and scientific points of view. A great deal of work concerning the initial breakdown of overvolted gaps has been published, and considerable understanding has been obtained, but there are still many unresolved questions involving the mechanisms which are important under a given set of conditions [1] .

Reports of fundamental studies of the spark phase of electrical breakdown are less numerous. Fast photographic techniques have been used to monitor the luminous spark channel in overvolted gaps [2]-[6], and the expanding shock front has been studied with Schlieren photography [2], [3], [7] . Temporally and spatially resolved spectroscopy has been used to determine electron density and temperature in hydrogen sparks [8], [9]. Theories of the spark channel and shock front expansion based on hydrodynamic arguments have been published by Drabkina [10], Braginskii [11], and Freeman and Craggs [7].

Information about the electron density in electrical sparks is important for proper understanding of the time evolution of the spark as well as the operating characteristics of the spark gap when used as a switching device. With these considerations in mind we have made a series of measurements of the temporally and spatially resolved electron densities in lasertriggered sparks in hydrogen. Electron density was determined by measurement of the Stark-broadened linewidth of emission lines of atomic hydrogen.

These studies differ from earlier work [2]-[9] primarily in that the spark current is lower and the spark results from the laser-triggered breakdown of an undervolted gap, whereas earlier studies involved conventional breakdown of overvolted

Manuscript received February 8, 1980; revised April 16, 1980. This work was sponsored in part by AFOSR under Contract F49620-79-C0191 and by the Research Corporation.

S. K. Dhali, P. F. Williams, and M. A. Gundersen are with the Department of Electrical Engineering, Texas Tech University, Lubbock, TX 79409.

R. J. Crumley was with the Department of Electrical Engineering, Texas Tech University, Lubbock, TX. He is now with Maxwell Laboratories, San Diego, CA 92123. gaps. We would expect the early stages of a laser-triggered spark to differ from the corresponding stages of an overvolted spark because the initial conditions for the spark undoubtedly are different. The later stages of the two types of sparks, on the other hand, are more likely to correspond.

The radial dependence of emission spectra is not directly determined from experimental measurements, but must be obtained through Abel transformation of the experimental data [12]. Earlier efforts [8], [9] at determining the radial dependence of the electron density in hydrogen sparks were hampered by the volume of data which must be processed numerically to transform a spectrum. As a consequence the electron density was determined from the ratio of intensities at line center and a fixed point in the wing. This method can produce erroneous results, however, because of selfabsorption of $\mathrm{H}_{\beta}$ emission or emission or absorption lines of trace impurity atoms in the gap. Even when low currents are used, we have seen evidence for the presence of both phenomena under some conditions.

Because our spectroscopy system is computer controlled, with digital data acquisition, we are in a position to Abel transform a complete spectrum on a point by point basis. As a check for spurious line broadening effects, the line shape of each transformed spectrum is compared with the theoretical Stark broadening profile. The limited capacity and speed of the minicomputer we use for data acquisition and analysis make the task somewhat tedious, but we feel the effort to be worthwhile in that it provides improved confidence in the results.

In this paper we present the first results for the temporally and spatially resolved electron density in laser-triggered hydrogen sparks. The Stark broadening technique is quite useful for determining electron densities in axially symmetric plasmas but we have encountered a few difficulties in the application of the technique to our problem, and these are discussed.

\section{Experimental Procedures}

Fig. 1 shows the equipment used for the experiments we describe. A spark gap consisting of two aluminum electrodes machined with a nearly constant-field profile was enclosed in a stainless steel vacuum/pressure cell. Triggering was accomplished with an $\mathrm{N}_{2}$ laser which delivered $10-\mathrm{ns} 4-\mathrm{mJ}$ pulses of $3361-\AA$ radiation. The beam was focused through a window and then a small hole in the upper gap electrode onto the lower electrode where it produced a small plasma "fireball" when tightly focused. For all experiments we report here the gap separation was $6.5 \mathrm{~mm}$. A charged coaxial cable system 


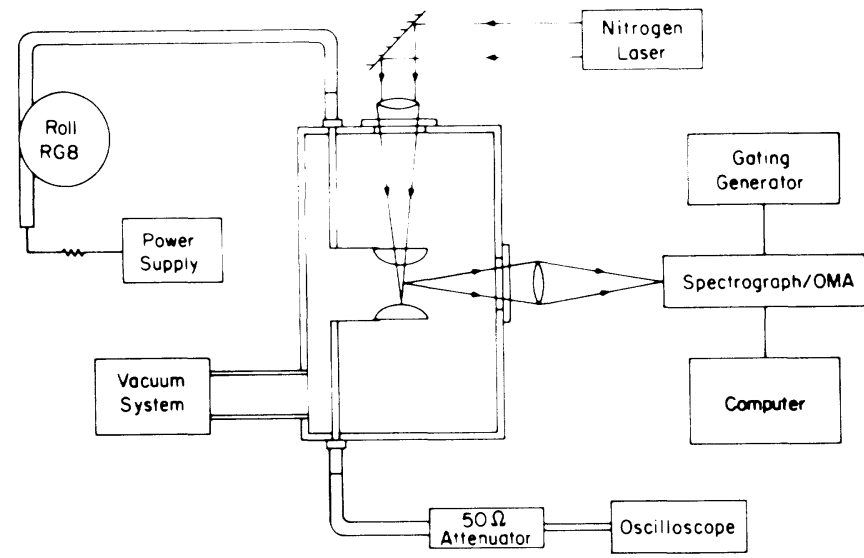

Fig. 1. Experimental arrangement.

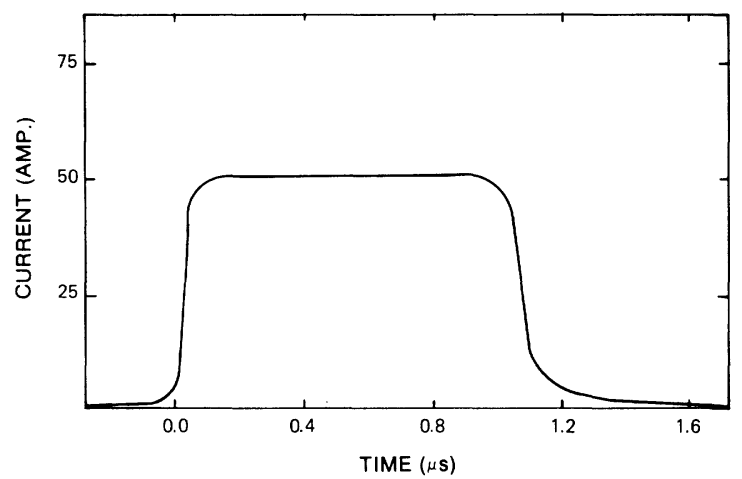

Fig. 2. Current pulse in laser triggered gap. $\mathrm{H}_{2}$ at 350 -torr, $5-\mathrm{kV}, 50-\Omega$ load.

was used to apply voltage to the gap. With careful matching of the 50- $\Omega$ load resistor, a clean current pulse (as shown in Fig. 2) with no reflection-induced afterpulse was obtained. Gap current was determined by measuring the voltage across the load resistor.

Optical access to the discharge for spectral analysis of the emission was provided by a second window transverse to the gap axis. An 0.5-m spectrograph coupled with a computercontrolled optical multichannel analyzer was used to obtain spectra of the discharge. Gating of the SIT detector of the analyzer provided temporal resolution down to $50 \mathrm{~ns}$. Spectra stored in the minicomputer could be transferred to a magnetic tape cartridge for later analysis. To obtain radially resolved spectra of the optical emission from the discharge, the arc image was focused on the entrance slit of the spectrograph with a photographic lens mounted on a precision translation stage. The slit and the discharge axis were parallel. The lateral dependence of the emission spectra was determined by sweeping the image across the entrance slit in small increments perpendicular to the discharge axis and obtaining and storing a spectrum for each position. The radial dependence of the spectra was obtained from this lateral data through the application of numerical Abel transform techniques. Finally, electron densities were determined by fitting the spectra thus obtained to theoretical line shape profiles.

\section{Abel Transform Techniques}

Assuming an optically thin axially symmetric discharge, the radial dependence of the emission coefficient $\epsilon(r)$ is related to

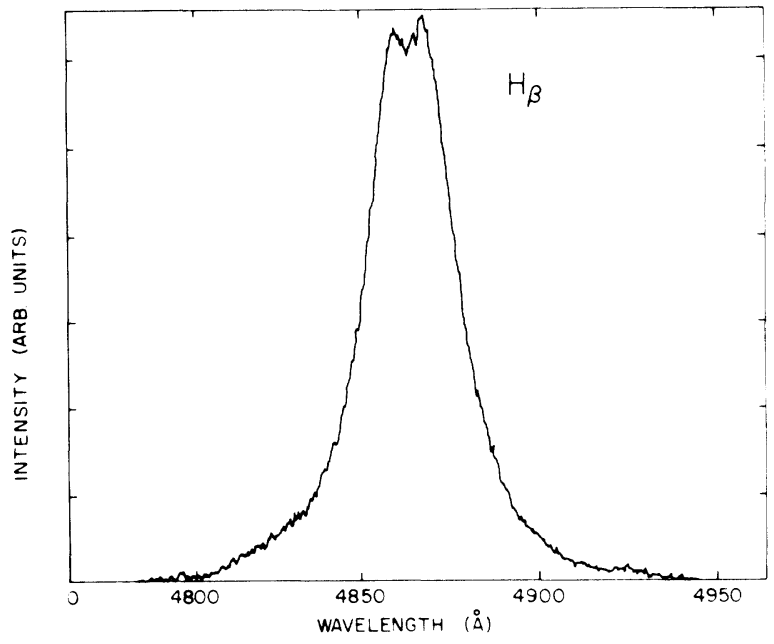

Fig. 3. Abel transformed line shape for $\mathrm{H}_{2}$ for $t=1.2 \mu \mathrm{s}, r=0 \mathrm{~mm}$.

the lateral intensity function $I(x)$ by [12]

$$
\epsilon(r)=-\frac{1}{\pi} \int_{r}^{r_{0}} \frac{I^{\prime}(x)}{\sqrt{x^{2}-r^{2}}} d x
$$

where $r_{0}$ is the radius of the discharge and the prime indicates differentiation. $I(x)$ is the experimentally determined quantity, and $\epsilon(r)$ is the desired function which is obtained by numerically evaluating the integral.

Because of difficulties involved with numerical differentiation, the integral was evaluated by first fitting the experimental $I(x)$ to a sum of Chebyshev polynomials, and then carrying out the differentiation and integration analytically. If the lateral intensity function at position $x_{j}$ and wavelength $\lambda_{k}$ is denoted by $I_{j k}$, and the radial emission coefficient at radius $r_{i}$ and wavelength $\lambda_{k}$ is denoted by $\epsilon_{i k}$, then as a result of this procedure $\epsilon_{i k}$ may be written as [13]

$$
\epsilon_{i k}=\sum_{j=1}^{N_{x}} C_{i j} I_{j k}
$$

where $N_{x}$ is the number of lateral positions and $C_{i j}$ is an $N_{r} \times N_{x}$ matrix ( $N_{r}$ is the number of radial positions) determined by the procedure just described.

This algorithm is relatively fast and efficient for calculating Abel transforms. Care must be taken, however, to ensure that the fitting procedure which approximates the actual lateral intensity functions by a polynomial does not introduce significant error. To this end, during the evaluation of (2) selected polynomial fits were plotted out along with the actual data to check on the accuracy of the procedure. For the data we report here the lateral intensity function was fit with a 20 th-order polynomial.

\section{Stark-Broadened Profiles}

Fig. 3 shows a typical Abel transformed spectrum of the $\mathrm{H}_{\beta}$ line of atomic hydrogen obtained with 400 torr of $\mathrm{H}_{2}$, after the current pulse. The electron density present in the spark channel at this point and time can be obtained directly from the width of the line [12], or by fitting the data to a theoretical line profile [14]. The latter method offers the advantages 
of being less sensitive to noise in the spectrum, and of providing a check on the line profile to ensure that Stark broadening was the dominant broadening mechanism. The electron densities we report here were obtained in this way.

Stark broadening profiles of a number of Balmer series lines of atomic hydrogen have been calculated by Griem [12]. To a good approximation these profiles may be expressed as a function of a reduced wavelength [12]

$$
\alpha=\left(\frac{4 \pi \cdot N_{e}}{3}\right)^{2 / 3} e \Delta \lambda
$$

where $N_{e}$ is the electron density and $\Delta \lambda$ is the wavelength displacement from line center. In order to fit the experimental data to the theoretical profile, log-log plots of both were made, with the theoretical profile plotted on a transparent sheet which could be positioned for the best fit with the experimental data. From the offset of the two plots the proportionality constant connecting $\alpha$ to $\Delta \lambda$ was determined, thereby defining the electron density $N_{e}$ through (3).

Besides the principal dependence on $\alpha$, the theoretical line profiles have a very weak further dependence on $N_{e}$ and $T$. We estimate the spark temperature to be about $20000 \mathrm{~K}$, and profiles corresponding to this value were used. Temperature variation of a factor of two makes only a slight change in the electron density determined in this way. Somewhat larger errors can result from the residual $N_{e}$ dependence of the profiles. The densities we report were obtained by determining the densities from profiles corresponding to two values of $N_{e}$ that bracket the true value and then interpolating between them.

Fig. 4 shows an example of the fit between the experimental data and the theoretical profile. We believe that the discrepancy at low intensities is primarily due to the strong nonlinearity of the SIT detector at low light levels. This nonlinearity represents a serious limitation on the usefulness of the SIT detector at low light levels. Presumably an additional stage of intensification on the detector would overcome the problem.

\section{EXPERIMENTAL RESULTS}

Electron density data obtained in the fashion we have described are shown in Fig. 5. The data were obtained with a 400-torr fill of hydrogen using the system described in a previous section and shown in Fig. 1. The electrode separation was $6.5 \mathrm{~mm}$ and the applied voltage was $5.0 \mathrm{kV}$. The time was measured from the point on the rising edge of the gap current pulse shown in Fig. 2. For all points the gating window was $100 \mathrm{~ns}$ wide. The spectrograph analyzed emission from a strip approximately $1.8 \mathrm{~mm}$ high located just above the cathode (the electrode struck by the laser). The entrance slit of the spectrograph provided resolution in the perpendicular direction of $0.075 \mathrm{~mm}$. The radial dependence of the $\mathrm{H}_{\beta}$ line emission intensity obtained under the same conditions is shown in Fig. 6.

The spark channel radius as determined from either the electron density or emission intensity data increases slowly during the spark phase of the discharge. This result is consistent with hydrodynamic theories which predict the rate of

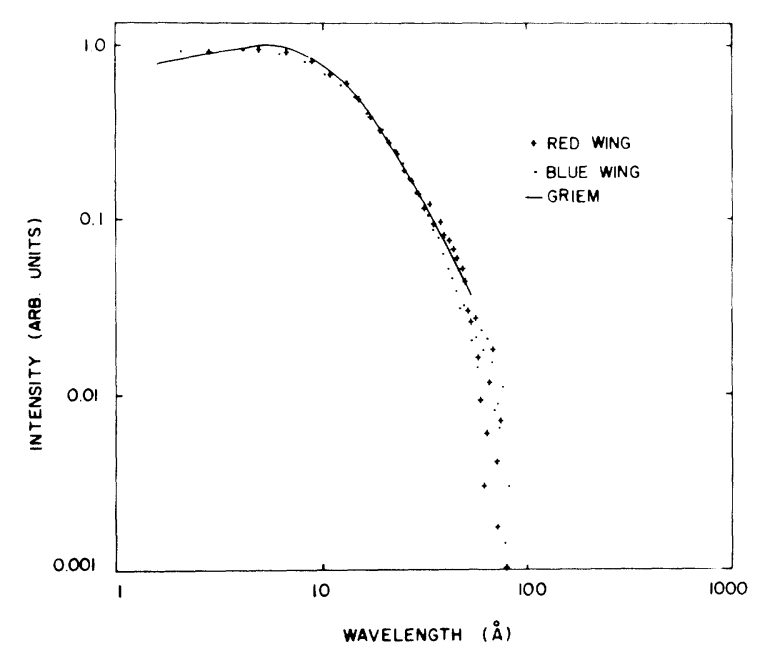

Fig. 4. Log-log plot of data in Fig. 3 with theoretical profile from Griem.

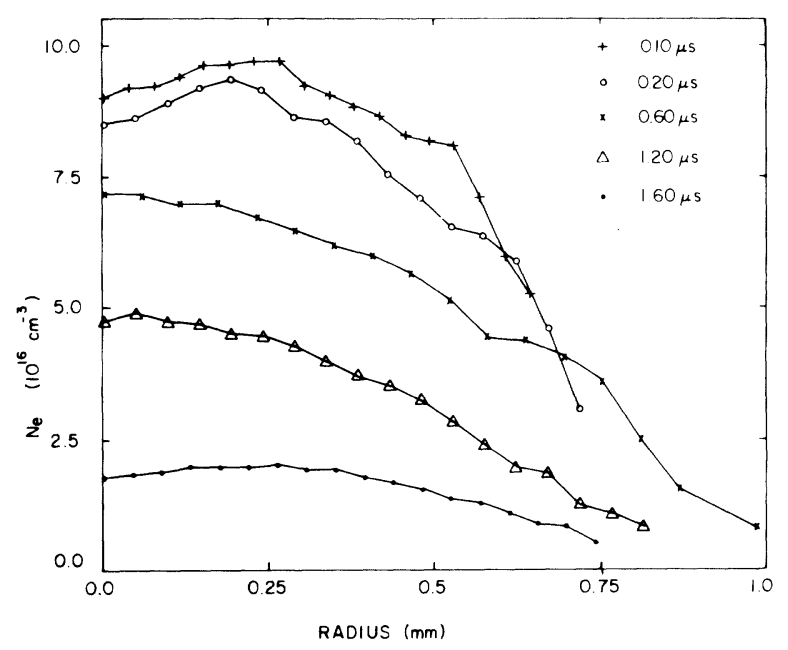

Fig. 5. Electron densities during and after current pulse from Stark broadening measurements. $\mathrm{H}_{2}$ at 400 torr.

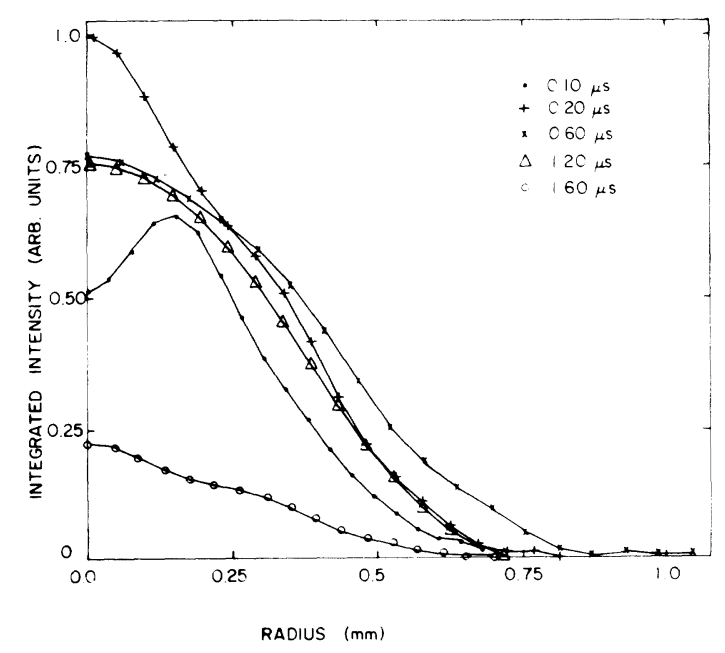

Fig. 6. Integrated emission intensities for the $H_{\beta}$ line under the same conditions as Fig. 5.

channel expansion to be a function of the electrical power deposited in the gap [11]. This power is a maximum during the rising edge of the current pulse and falls to a low value during the spark phase due to the low voltage drop across the 
gap. For times after the spark has extinguished, the channel radius collapses rapidly, indicating the need for a thermal conduction term in any hydrodynamic analysis of the spark.

Tholl has reported the results of extensive measurements of spatially and temporally resolved electron density and temperature in overvolted hydrogen sparks [8], [9]. The spark current in these measurements was much higher than in our experiments. The primary difference between the results we report here and those of Tholl is in the form of the radial dependence of the electron density. In our data the valley at small radii is very much less prominent than in the data of Tholl. At this time it is not known if this difference results from the much lower current densities or from the lasertriggering of our discharge. Experiments involving higher current discharges are currently underway.

We thank R. D. Bengston, E. E. Kunhardt, W. G. Braun, and A. Garscadden for helpful discussions.

\section{REFERENCES}

[1] See, for example, Electrical Breakdown of Gases, J. M. Meek and J. D. Craggs, Eds. New York: Wiley, 1978.

[2] J. Koppitz, "Die radiale und axiale Entwicklung des Leuchtens im Funkenkanel, untersucht mit einer Wischkamera," Z. Naturforsh., vol. 22a, pp. 1089-1097, 1967.

[3] V. F. Egorova, V. L. Isanko, A. A. Mak, and A. L. Sadykova,
"Distribution of temperature and electron density is a spark discharge canal," Sov. Phys.-Tech. Phys., vol. 7, pp. 242-247, Sept. 1962.

[4] A. A. Doran and J. Meyer, "Photographic and oscillographic investigations of spark discharges in hydrogen," Brit. J. Appl. Phys., vol. 18, pp. 793-799, 1967.

[5] M. C. Cavenor and J. Meyer, "The development of spark discharges in hydrogen," Aust. J. Phys., vol. 22, pp. 155-167, 1969.

[6] M. Kekez, M. R. Barrault, and J. D. Craggs, "Spark channel formation," J. Phys. D: Appl. Phys., vol. 3, pp. 1886-1896, 1970.

[7] R. A. Freeman and J. D. Craggs, "Shock waves from spark discharges," Brit. J. Appl. Phys., vol. 2, pp. 421-427, 1969.

[8] H. Tholl, "Spektroskopische Messung des Zeitlichen Verlaufs den radialen Dichte und Temperaturverteilung in einem Wasserstoffunken," Z. Naturforsh., vol. 22a, pp. 1068-1088, 1967.

[9] H. Martinen and H. Tholl, "Untersuchung der Temperatur und der Expansion von Funkenkanälen in $\mathrm{H}_{2}$ bei variabler Energiezufuhr," $Z$. Naturforsh., vol. 25a, pp. 436-439, 1970.

[10] S. I. Drabkina, "The theory of the development of the channel in the spark discharge," Zh. Eksp. Teor. Fiz., Pisma Red., vol. 21, pp. 473-493, 1951.

[11] S. I. Braginskii, "Theory of the development of a spark channel," Sov. Phys. JETP, vol. 34, pp. 1068-1074, 1958.

[12] H. R. Griem, Plasma Spectroscopy. New York: McGraw-Hill, 1964.

[13] R. J. Crumley, "Electron densities in laser-triggered spark gap discharges," unpublished thesis, Texas Tech University.

[14] R. D. Bengston and G. R. Chester, "Stark broadening of Balmer lines in the density range $(2-8) \times 10^{14} \mathrm{~cm}^{-3}$," Phys. Rev. A, vol. 13, pp. 1762-1771, May, 1976. 